\title{
Characterization of a Combined CARS and Interferometric Rayleigh Scattering System
}

\author{
S. A. Tedder, College of William and Mary, Williamsburg, VA 23669 \\ D. Bivolaru ${ }^{\dagger}$, P. M. Danehy ${ }^{\ddagger}$, NASA Langley Research Center, Hampton, VA 23681 \\ M. C. Weikl ${ }^{\S}$, F. Beyrau", T. Seeger ${ }^{\Uparrow}$, Universität Erlangen-Nürnberg, Erlangen, Germany \\ A. D. Cutler ${ }^{* *}$, The George Washington University, Newport News, VA 23602
}

\begin{abstract}
This paper describes the characterization of a combined Coherent anti-Stokes Raman Spectroscopy and Interferometric Rayleigh Scattering (CARS-IRS) system by reporting the accuracy and precision of the measurements of temperature, species mole fraction of $\mathrm{N}_{2}, \mathrm{O}_{2}$, and $\mathrm{H}_{2}$, and two-components of velocity. A near-adiabatic $\mathrm{H}_{2}$-air Hencken burner flame was used to provide known properties for measurements made with the system. The measurement system is also demonstrated in a small-scale Mach $1.6 \mathbf{H}_{2}$-air combustionheated supersonic jet with a co-flow of $\mathbf{H}_{2}$. The system is found to have a precision that is sufficient to resolve fluctuations of flow properties in the mixing layer of the jet.
\end{abstract}

\section{Introduction}

$\mathbf{T}$ ime-resolved measurements are desirable to investigate unsteady and turbulent flows. Non-intrusive multiproperty optical methods have been applied to high-speed flows and combustion experiments using Dual-Pump CARS for temperature and species mole fraction, ${ }^{1,2,3}$ and Rayleigh scattering for temperature, density, and velocity. ${ }^{4,5,6,7}$ These measurements serve as good test cases for evaluating or developing CFD codes. When multiple flow properties are measured simultaneously, correlations can be used to obtain a deeper understanding of combusting and turbulence flows. For example, correlations can be used to evaluate parameters in new turbulence models. In Reference 8, we reported the combination, for the first time, of a dual-pump CARS system with an interferometric Rayleigh scattering optical system (CARS-IRS) to provide time-resolved simultaneous measurement of temperature, species concentration, and multiple components of velocity in gaseous media.

The CARS-IRS system has been developed mainly to study reacting flows. Two different sets of supersonic reacting jet hardware have been constructed: a lab-scale version ${ }^{9}$ and a more than 6 times larger version to be used in a NASA test facility. The purpose of the smaller apparatus is both to verify the operability of the jet design and to validate the measurement techniques in a low-cost laboratory setting. The larger-scale experiment is needed to generate data sets for the CFD modelers by adequately resolving the spatial scales of the flow.

Since our first reporting of CARS-IRS data, ${ }^{8}$ the CARS signal strength was increased to improve the signal-tonoise ratio at high temperatures, allowing for higher accuracy and precision at those temperatures. The spectral resolution of the CARS system was also improved to allow higher sensitivity to temperatures near room temperature. Furthermore, improvements were made to the Rayleigh scattering system and software analysis to reduce the size, and improve the performance and reliability of the system.

Further preparation for the large-scale experiment included measurements in a near-adiabatic, laminar flat flame Hencken burner. This high-temperature source provides a steady, known (easily calculable) temperature and species concentration, and a near-zero velocity, allowing for determination of accuracy and precision of the system. To verify the performance of the system in a noisy environment, measurements were taken in the small-scale supersonic reacting jet flow. A measurement example showing the capability of the system to measure in the highenthalpy Mach 1.6 supersonic flow is included.

\footnotetext{
${ }^{*}$ Research Assistant, College of William and Mary, Williamsburg, VA 23669, AIAA Member

${ }^{\dagger}$ NASA Postdoctoral Fellow, Advanced Sensing and Optical Measurement Branch, MS 493, AIAA Member

* Research Scientist, Advanced Sensing and Optical Measurement Branch, MS 493, AIAA Associate Fellow

$\S$ Research Assistant, Universität Erlangen-Nürnberg, Erlangen, Germany

" Research Scientist, Universität Erlangen-Nürnberg, Erlangen, Germany

*** Professor, The George Washington University, Newport News, VA 23602, AIAA Associate Fellow
} 


\section{Experimental Setup}

The experimental arrangement of the system is described in detail in Reference 8 . Figure 1 shows the combining and receiving optics at the measurement volume. For the measurement of temperature and the absolute mole fractions of $\mathrm{N}_{2}, \mathrm{O}_{2}$, and $\mathrm{H}_{2}$ we use a dual-pump CARS method. ${ }^{1,2,3}$ The system uses spectrally-narrow green (injection seeded Nd:YAG at $532 \mathrm{~nm}$ ) and narrow-band yellow (dye laser at $552.75 \mathrm{~nm}$ ) laser pump beams and a spectrally broad red laser (dye laser centered at $604 \mathrm{~nm}$ ) beam as the Stokes beam. The $850 \mathrm{~mJ} /$ pulse energy of the Nd:YAG laser is split in three ways. About $350 \mathrm{~mJ}$ of the energy is used for the CARS green pump beam and for Rayleigh scattering. A pulse stretcher ${ }^{8}$ is used in this beam optical path so that the energy is delivered to the probe volume as a series of three or more separated pulses to avoid laser-induced breakdown and saturation effects in CARS. Another $250 \mathrm{~mJ}$ is used for pumping the broadband dye laser that uses Rhodamine 640 laser dye diluted in methanol. The rest of the Nd:YAG laser energy is used to pump the tunable narrowband dye laser. The beam energies at the measurement volume varied during experiments in the range of 150 to $200 \mathrm{~mJ} /$ pulse for the green, 2 to $10 \mathrm{~mJ} /$ pulse for the red, and 5 to $15 \mathrm{~mJ} /$ pulse for the yellow laser beam.

The beams are combined at the focusing point of a spherical lens $L_{1}$ (focal length of $410 \mathrm{~mm}$ ) in a folded BOXCARS geometry ${ }^{10}$ generating a measurement volume of about $1.5 \mathrm{~mm} \times 0.2 \mathrm{~mm} \times 0.2 \mathrm{~mm}$. The frequency difference between the green and red beams corresponds to the vibrational Raman shift of $\mathrm{N}_{2}$. The frequency of the yellow pump beam is chosen so that the frequency difference between the yellow and red beams equals the vibrational Raman shift of $\mathrm{O}_{2}$. Pure-rotational $\mathrm{H}_{2}$ Raman transitions are also present in both spectral regions. The input beams plus the coherent blue signal beam at $491 \mathrm{~nm}$ are collected and collimated by another spherical lens $\mathrm{L}_{2}$ with the same focal length as $\mathrm{L}_{1}$, after which the pumps and Stokes beams are blocked with the beam dump BD. After passing through an interference filter that rejects stray light, the signal beam is focused by two "crossed" cylindrical lenses on the input slit of a one-meter spectrometer. The CARS signal, which is a spectrally broad blue beam that contains $\mathrm{N}_{2}, \mathrm{O}_{2}$ and $\mathrm{H}_{2}$ spectra, is dispersed by a spectrometer and recorded by a CCD camera. The shape of these spectra provides information on the temperature while the relative intensities of the different molecules' spectra provide a measure of the relative mole fractions. The spectrum is fit with a theoretical model to determine the temperature and mole fractions. For further details of the analysis method see Reference 8 .

The interferometric Rayleigh scattering measurement system, is similar to earlier versions which are described in References 8 and 11. The same seeded green laser beam employed for CARS is used as a narrow-band light source for the Rayleigh system. The receiving optics collect Rayleigh scattered light from the green beam at the measurement volume while preserving the scattering angle information. The light collected by the lens $\mathrm{L}_{3}$ in the direction of $\boldsymbol{k}_{s 1}$ (perpendicular to both the laser beam axis and the flow axis) has a Doppler shift frequency given by $\Delta v_{1}=\left(\boldsymbol{k}_{s 1}-\boldsymbol{k}_{0}\right) \cdot \boldsymbol{V}$, where $\boldsymbol{k}_{0}$ is the input laser beam wave vector, $\boldsymbol{k}_{\mathrm{s} 1}$ is the wave vector of the scattered light, and $\boldsymbol{V}$ is the velocity vector. The direction of the vector $\left(\boldsymbol{k}_{s 1}-\boldsymbol{k}_{0}\right)$ gives the direction of the velocity component being measured, defined as $V_{1}$. Given the orientation of this vector relative to the burner or jet exit plane, for measurements near the jet axis or when scanning through the jet axis along the vector $\mathrm{V}_{1}$, this velocity vector is the radial component of velocity, $\boldsymbol{V}_{r}$ of the flow. For measurements in other locations, the vector $\mathrm{V}_{1}$ contains also the tangential velocity component $\boldsymbol{V}_{\theta}$. To measure the second component of velocity $\boldsymbol{V}_{z}$ in the axial direction of the flow, scattered light is collected by lens $\mathrm{L}_{4}$ in the direction of the wave vector $\boldsymbol{k}_{s 2}$ in a plane (containing $\boldsymbol{k}_{s 1}$ ) perpendicular to the plane defined by the vectors $\boldsymbol{k}_{s l}$ and $\boldsymbol{k}_{0}$. The angle between $\boldsymbol{k}_{s 2}$ and $\boldsymbol{k}_{s l}$ is $32.5 \pm 0.5^{\circ}$. The 
direction of $\left(\boldsymbol{k}_{s 2}-\boldsymbol{k}_{0}\right)$ vector gives the direction of the velocity vector being measured, defined as $\mathrm{V}_{2}$. This vector contains information from both components of velocity $\boldsymbol{V}_{r}$ and $\boldsymbol{V}_{z}$. Therefore the velocity vectors $\mathrm{V}_{1}$ and $\mathrm{V}_{2}$ contain the components $\boldsymbol{V}_{r}$ and $\boldsymbol{V}_{\theta}$, and $\boldsymbol{V}_{r}, \boldsymbol{V}_{\theta}$, and $\boldsymbol{V}_{z}$, respectively. The light from these two directions is combined into a single collimated beam, filtered to remove the scattered light from the yellow and the red CARS beams, and passed through a solid Fabry-Perot etalon. An electron multiplication gain CCD camera records the interference fringe pattern, which is formed at the output of the etalon. The etalon used in these experiments has a free spectral range of $8.33 \mathrm{GHz}$ and a reflectivity finesse of 22. Both the CARS spectra and the Rayleigh interferograms are acquired simultaneously by synchronizing the cameras with the green laser Q-switch pulse at $20 \mathrm{~Hz}$.

Modifications were made to the CARS system described in Reference 8 in order to increase the accuracy and precision. The 1200 line $/ \mathrm{mm}$ grating was replaced with a 2400 line $/ \mathrm{mm}$ grating to increase the resolution of the CARS spectra. The CARS signal was increased in intensity by increasing the energy output, improving the beam quality of the narrowband and broadband dye lasers, and by improving the spatial and temporal overlaps at the measurement volume.

Single-shot measurements were performed in a Hencken burner ${ }^{12}$ to characterize the system. This burner provides a relatively uniform and well-understood environment in which to measure temperature and species concentration. The axisymmetric laboratory-scale supersonic burner ${ }^{9}$ consists of a combustion chamber and a central convergent-divergent nozzle with an exit diameter of $10 \mathrm{~mm}$. A coflow nozzle generates an annular flow region between the central jet and the ambient air. Reactants are delivered to the burner and coflow to produce a highenthalpy vitiated jet at Mach 1.6. This device is being used to develop the test matrix of future large-scale experiments ${ }^{9}$ and to verify the CARS-IRS measurement techniques. However, some of the fluid dynamical length scales of the flow are too small to be resolved by the CARS measurement volume, which is about $1.5 \mathrm{~mm}$ long.

\section{A. Single shot CARS Spectra}

Figure 2 shows single-shot spectra taken in a Hencken burner for two different equivalence ratios. With increasing temperature the $\mathrm{N}_{2}$ "hot band" near $2290 \mathrm{~cm}^{-1}$ increases while the overall CARS signal intensity decreases. This lower overall intensity results in a decreased signal-to-noise ratio of the data, predominantly at the edges of the spectrum. This can be seen in Figure 2 from a) to b). Also in Figure 2, the mole fractions of $\mathrm{N}_{2}$ and $\mathrm{O}_{2}$ decrease in response to an increase in $\mathrm{H}_{2}$ (increase in equivalence ratio). Comparing these spectra to spectra shown in Reference 13 the spectral resolution has increased showing better resolved rotational lines in the peaks corresponding to $\mathrm{N}_{2}$ and $\mathrm{O}_{2}$. However, the current spectra show increased noise at the edges. This is probably caused by the $30 \%$ decrease in the FWHM of the nonresonant spectrum produced by the new broadband dye laser. Very little energy probes the edge of the broadband spectrum, resulting in low signal intensity in the vicinity of the $\mathrm{H}_{2}$ lines. During data processing ${ }^{8}$ the resonant spectrum is divided by the nonresonant spectrum. The edge of the nonresonant spectrum, which is low in intensity, amplifies the noise. The $\mathrm{H}_{2}$ lines $\mathrm{S}(5)$ and $\mathrm{S}(6)$ are located at exactly this position, which leads to a lower accuracy for concentration measurement compared to our past work. ${ }^{13}$

\section{Results}

a)

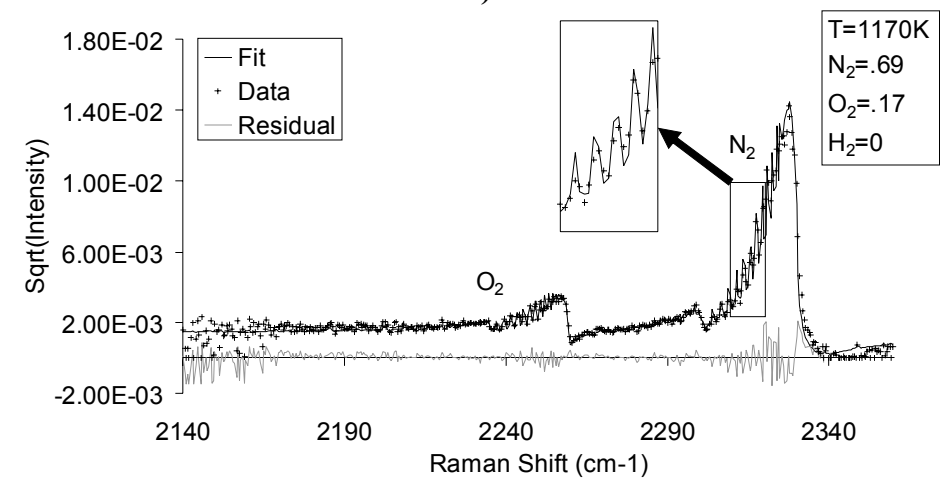

b)

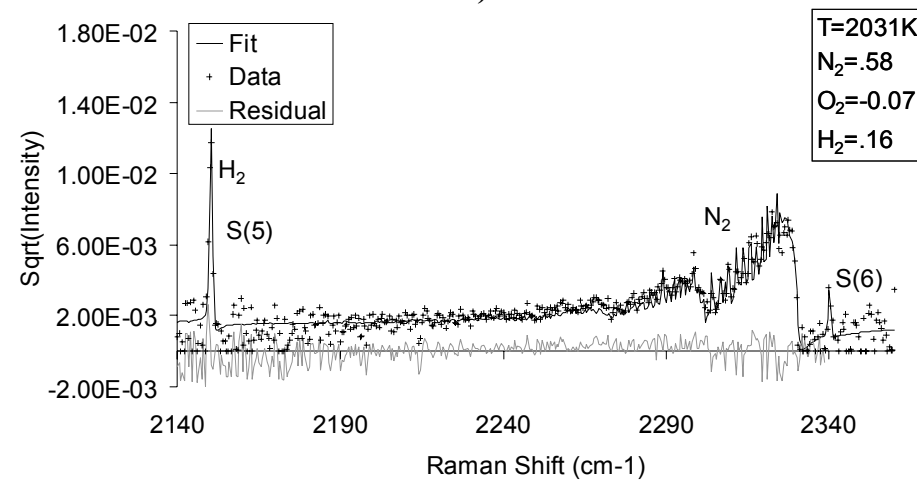

Figure 2. Single shot CARS Spectra in a $\mathrm{H}_{2}$-air laminar flame at different equivalence ratios $(\Phi)$, a) $\Phi=0.3$, b) $\Phi=1.8$ 


\section{B. CARS-IRS System Characterization}

A series of measurements to determine the accuracy and precision of the system were performed using a Hencken burner flame. ${ }^{12}$ Computed values of temperature and composition over a range of fuel-to-air ratios (equivalence ratios) were used for this comparison. The Hencken burner also provided a near-zero velocity flow to characterize the IRS velocity measurement system. In Figure 3 the measured temperatures, shown as diamonds, are compared to theoretical temperatures, shown by a solid line. The error bars represent \pm 1 standard deviation of the 400 acquisitions taken at each equivalence ratio. One standard deviation is used as a benchmark for the measurement of the instrument's precision. The standard deviation increases with temperature from $20 \mathrm{~K}$ at $300 \mathrm{~K}$ to typically $50-60 \mathrm{~K}$ for equivalence ratios less than one to $60-90 \mathrm{~K}$ for equivalence ratios greater than or equal to one. This results in a relative standard deviation that decreases with temperature as the hot band of the $\mathrm{N}_{2}$ increases providing a temperature-sensitive shape to fit. At temperatures higher than $1600 \mathrm{~K}$ the development of the hot band no longer improves the relative standard deviation and the relative precision remains constant.

The measurement at an equivalence ratio of 1.0 and 1.5 do not follow the precision trends of the rest of the data set; a possible explanation is an error in the fitting procedure.

At 1.5 almost half of the 400 acquisitions fit to a temperature near $2000 \mathrm{~K}$ and the other half fit to a temperature near $2300 \mathrm{~K}$ with only a few fitting to values in between. At equivalence ratio 1.0 there is a similar bifurcation but it is more subtle. These bifurcations of the temperature measurements are unphysical. It is probably some anomaly of our spectral fitting techniques that has arisen as a result of our fitting more highly resolved spectra than in our previous work. This issue will be resolved before applying our method in the study of reacting flows.

Figure 4 shows mole fractions versus equivalence ratios for $\mathrm{N}_{2}, \mathrm{O}_{2}$, and $\mathrm{H}_{2}$. The solid lines represent theoretical values for mole fraction, determined from the gas flow rates, and the points represent the values measured by CARS. For equivalence ratios less than one the $\mathrm{O}_{2}$ mole fractions are typically higher than the theory by $\sim 0.03$ and then are well below zero for higher mole fractions, when they should be zero. This is another unphysical result that will need to be corrected through improved experimentation and/or data analysis. For equivalence ratios greater than one, the $\mathrm{H}_{2}$ mole fractions are consistently low by $\sim 0.05$, qualitatively similar to the results in Reference 13 . Further comparing these Hencken burner results with results presented in Reference 13, the temperatures in the current work are less precise at equivalence ratios higher than one. This difference is probably a result of the decrease in FWHM of the broadband dye spectra, mentioned above. These issues are exacerbated at equivalence ratios greater than one where $\mathrm{H}_{2}$ is present (recall that the $\mathrm{H}_{2}$ lines lie in the wings of the CARS spectra).

As mentioned earlier, velocity measurements were performed simultaneously with CARS. The standard deviation of velocity calculated from 400 consecutively recorded spectra (98\% of the data fitted successfully) is shown in Fig. 5 as a function of the equivalence ratio (for which temperature and composition is changing significantly). As expected the standard deviation is seen to increase with equivalence ratio as the temperature increases and the density decreases, resulting in lower Rayleigh signal intensities. A smaller standard deviation is found for the radial component $\boldsymbol{V}_{r}$ because the component $\boldsymbol{V}_{z}$ is calculated from $\mathrm{V}_{1}$ and $\mathrm{V}_{2}$ and thus contains the 
combined error of these two measurements. The standard deviation, $\sigma$, of $\boldsymbol{V}_{r}$ (black squares) and $V_{2}$ (red triangles) is smaller than $27 \mathrm{~m} / \mathrm{sec}$ at the highest temperature with a minimum of $8 \mathrm{~m} / \mathrm{sec}$ in stagnant air. The standard deviation of the calculated axial velocity $\boldsymbol{V}_{z}$ (red circles) is less than 57 $\mathrm{m} / \mathrm{sec}$ in the whole range of measurements.

To quantify the systematic errors of the velocity measurement, we assume that both components of velocity in the burner are near zero. This is based on the observation that the estimated radial velocity and the maximum axial velocity are smaller than or comparable with the errors of the measurement (almost zero for radial velocity and less than $10 \mathrm{~m} / \mathrm{s}$ in the case of the axial velocity). We define a velocity offset $\mathrm{V}_{\text {offset }}$ as the apparent measured value of the velocity in stagnant air or near-zero velocity gases. This offset is caused by the noncoincidence of the reference fringe pattern used as frequency markers in the interferogram image and the Rayleigh scattering with zero Doppler shifts. The existence of a velocity bias in an interferogram containing multiple fringe patterns ${ }^{8}$ is consistent with similar observations by other researchers in this field $^{14}$. The offset velocity values $\left(\mathrm{V}_{\text {offset }}\right)$ reported here contains both the measurement offset and the actual velocity of the flow (considered zero or very close to zero). For velocity components measured directly, $\mathrm{V}_{1}$, and $V_{2}$, the velocity offset varies from minus $150 \mathrm{~m} / \mathrm{sec}$ in room air to $33 \mathrm{~m} / \mathrm{sec}$ at equivalence ratio of one (where the temperature is $2380 \mathrm{~K}$ ). We believe that the velocity-offset variation with increasing temperature is mostly caused by optical alignment, which was changed by re-adjustments during the course of this particular experiment. If $\mathrm{V}_{\text {offset }}$ is determined for a particular alignment of the system it can be used as a calibration parameter in calculating Doppler shifts measured subsequently.

This system characterization in the Hencken burner allows noise from the instrument to be distinguished from variations in the flow properties, since the flow is relatively quiescent.

\section{Supersonic Reacting Jet}

Measurements to demonstrate the capability of the system were performed on the laboratory-scale version of the supersonic reacting jet described in Reference 9. These experiments provided a challenging noisy and turbulent environment, with a noise level up to $140 \mathrm{~dB}$ at $2 \mathrm{~m}$ away from the test jet. Data sets of 1000 consecutive spectra of CARS and Rayleigh scattering are used for this evaluation. Figure 6 is a comparison of measurements of temperatures at $\mathrm{z}=70 \mathrm{~mm}$ downstream of the nozzle. Temperatures at the center of the jet are shown in blue histogram bins. They are less scattered compared to temperatures measured $12 \mathrm{~mm}$ from the center of the jet (filled black bins) where mixing of cold ambient air and hot vitiated air, as well as combustion, is occurring. This figure demonstrates the capability of the system to distinguish between, and to quantify, steady and turbulent flow. The histogram has bins of $100 \mathrm{~K}$ to reflect the precision of the CARS instrument determined in the Hencken burner.

Histograms representing single-point measurements of velocity on the axis of the jet using the IRS system are shown in Fig. 7. The velocity components $\boldsymbol{V}_{\boldsymbol{r}}=\mathrm{V}_{1}$ (black) and $\mathrm{V}_{2}$ (red) are measured directly, while the axial component $V_{z}$ (blue) is calculated from $V_{1}$ and $V_{2}$. The histograms have bins of $15 \mathrm{~m} / \mathrm{sec}$ for directly measured 
velocity components and $45 \mathrm{~m} / \mathrm{sec}$ for the calculated ones. In Fig. 7a, a measurement at $\mathrm{z}=10 \mathrm{~mm}$, the non-zero radial velocity $\boldsymbol{V}_{\boldsymbol{r}}$ is attributed to a slightly off-axis measurement. The measurement at $\mathrm{z}=70$ $\mathrm{mm}$ presented in Fig. $7 \mathrm{~b}$ shows a broader velocity distribution than in Fig.7a. The large fluctuation of $\boldsymbol{V}_{z}$ about the mean (of more than $25 \%$ ) and the asymmetry in the velocity distribution more accentuated for the $V_{2}$ component (on higher velocities side) are probably caused both by errors in the fitting technique of Rayleigh spectra and turbulence effects in the jet.

Fig. 8 shows correlations of fluctuations about the mean of the directly measured velocity components $V_{1}{ }^{\prime}=\boldsymbol{V}_{\boldsymbol{r}}{ }^{\prime}=$ $\mathrm{V}_{1}-\operatorname{mean}\left(\mathrm{V}_{1}\right)$ and $\mathrm{V}_{2}{ }^{\prime}=\mathrm{V}_{2}-\operatorname{mean}\left(\mathrm{V}_{2}\right)$ and temperature $\mathrm{T}^{\prime}=\mathrm{T}_{\mathrm{CARS}}-\operatorname{mean}\left(\mathrm{T}_{\mathrm{CARS}}\right)$ measured on the axis of the jet at $z=70$ $\mathrm{mm}$ from the nozzle exit (seven nozzle diameters). The precision (one standard deviation) at these conditions is expected, from the Hencken burner data, to be about $54 \mathrm{~K}$ for temperature and about $15 \mathrm{~m} / \mathrm{sec}$ for velocity. The circular pattern of the data scattered having more than 5 standard deviations shows that there are fluctuations in the jet that are greater than the measurement precision determined in the Hencken burner. But, there is no correlation between the simultaneously measured velocity and temperature at this location (a linear or curved trend would have indicated a correlation). On the axis, the symmetry of the jet requires that the correlation of $\boldsymbol{V}_{r}^{\prime}$ with $\mathrm{T}^{\prime}$ to be zero, on average, but there should be a small correlation between $\mathrm{V}_{2}{ }^{\prime}$ and $\mathrm{T}^{\prime}$. It is possible that in the harsh conditions of the reacting jet flow (optical steering of laser beams and acoustically noisy environment), the actual measurement precisions may not be as good as in the Hencken burner. So, the absence of correlation in Figure 8 could be because the turbulence contribution to fluctuations on the flow axis is small compared to the measurement precision. At this point in the flow the mixing layer has not yet reached the flow axis so it is relatively quiescent. Another possible reason for the lack of correlation is that the CARS and IRS measurement volumes may be probing slightly different (uncorrelated) spatial locations.

\section{Discussion and Conclusions}

By taking measurements in a similar environment to the planned experiment and characterizing the system, an assessment of how to increase the accuracy and precision of the system was obtained. A broader nonresonant spectrum (defined by the Stokes laser) to increase the signal-to-noise level at the edges of the CARS spectra would increase the precision of temperature and precision and accuracy of species mole fractions. Improving the fitting algorithm in the fitting codes would also increase both accuracy and precision of all measurements made with 
CARS. Because the nonresonant spectrum can change with time, measurements of its shape in real time may help for more accurate fitting. The CARS probe volume is nearly ellipsoidal in form with a long dimension of $1.5 \mathrm{~mm}$. It is wider in the direction of the laser propagation than perpendicular to it. For high resolution measurements in the full-scale supersonic reacting jet, measurement location scans should be made in the direction perpendicular to the laser propagation direction.

Raman rotational transitions $\mathrm{S}(5)$ and $\mathrm{S}(6)$ are used to probe $\mathrm{H}_{2}$. At room temperature these transitions have signal intensities that are below the noise level because the population in the corresponding energy levels is very low; most of the $\mathrm{H}_{2}$ molecules reside in the lowest few energy states. This limits the ability of CARS to measure temperature, for example, room temperature in pure $\mathrm{H}_{2}$ using the present method. When no $\mathrm{N}_{2}$ is present (for example in the pure- $\mathrm{H}_{2}$ co-flow of the jet) and the $\mathrm{H}_{2}$ lines have very low signal intensity (or are not present) it is not possible to accurately determine temperature or concentration from these lines. A possible solution to this problem is to develop a more complicated measurement system that probes lower rotational quantum number $\mathrm{H}_{2}$ transitions. Another solution would be to add a small amount (for example, a few percent) of $\mathrm{N}_{2}$ to the $\mathrm{H}_{2}$ co-flow. Temperatures could be determined from the strong $\mathrm{N}_{2}$ spectra and then $\mathrm{H}_{2}$ concentrations could be determined from the intensity of the $\mathrm{H}_{2}$ line(s).

The accuracy and precision of data taken with this CARS-IRS system depends on the signal-to-noise ratio. New strategies that could reduce further the measurements uncertainty and include the velocity offset as a calibration factor of the instrument will be investigated. For example the measurement of the axial velocity $\boldsymbol{V}_{z}$ could be improved by measuring the component directly by using a different geometrical arrangement of laser beams and detection optics. This can be achieved by rotating the measurement system or by re-orientating the flow being investigated. More convenient may be the addition of another green beam focused at the same measurement volume from another direction. But, collecting additional Rayleigh scattering may complicate the combining optics and their adjustment (of three measurement volumes), the interferogram analysis of the Rayleigh spectra, and may limit the region where the measurements can be performed.

Using a Hencken Burner to measure the precision of the CARS-IRS system provided distinction between errors originating from the measurement technique and variations originating from the flow being investigated. Results from the small-scale reacting jet demonstrate that the system is capable of making measurements in supersonic combustion flows and can resolve variations in the turbulent flow field.

\section{Acknowledgements}

This work was supported by the NASA Fundamental Aeronautics, Hypersonics Program and also by Mr. George Rumford, program manager of the Defense Test Resource Management Center's (DTRMC) Test and Evaluation/Science and Technology (T\&E/S\&T) program, under the Hypersonic Test focus area. We also thank to Lloyd G. Wilson for installing and operating the Hencken Burner and the laboratory-scale reacting supersonic jet.

\section{References}

\footnotetext{
${ }^{1}$ Danehy, P.M., O’Byrne, S., Cutler, A.D., and Rodriguez, C. G., "Coherent anti-Stokes Raman Scattering (CARS) as a probe for supersonic hydrogen-fuel/air mixing," JANNAF APS/CS/PSHS/MSS Joint Meeting, Colorado Springs, Colorado, December 1-5, 2003.

${ }^{2}$ O’Byrne, S., Danehy, P.M., Cutler, A.D., "Dual-Pump CARS Thermometry and Species Concentration Measurements in a Supersonic Combustor," 42 ${ }^{\text {nd }}$ Aerospace Sciences Meeting and Exhibit, Reno, NV, January 5-8, 2004.

${ }^{3}$ Danehy, P. M., R. DeLoach, R., Cutler, A. D., "Application of Modern Design of Experiments to CARS Thermometry in a Supersonic Combustor," $22^{\text {nd }}$ AIAA Aerodynamic Measurement Technology and Ground Testing Conference, AIAA Paper 2002-2914, June 2002.

${ }^{4}$ Miles, B., Richard, Lempert, R., Walter and Forkey, N. Joseph, "Laser Rayleigh Scattering," Measurement Science and Technology, Vol. 12, pp. 33-51, 2001.

${ }^{5}$ Seasholtz, R. G., Zupanc, F. J. and Schneider, S. J., "Spectrally Resolved Rayleigh Scattering Diagnostics for HydrogenOxygen Rocket Plume Studies, ” J. Propulsion and Power, Vol. 8, No. 5, 1992, pp. 935-942.

${ }^{6}$ Seasholtz, R. G., Buggele, A. E. and Reeder, M., "Instantaneous Measurements in a Supersonic Wind tunnel Using Spectrally Resolved Rayleigh Scattering," Proceedings of the International Symposium on Optical Science, Engineering and Instrumentation, Society of Photo-Optical Instrumentation Engineers, Bellingham, WA, 1995.

${ }^{7}$ Bivolaru, D., Ötügen, M. V., Tzes, A. and Papadopoulos, G., "Image Processing for Interferometric Mie and Rayleigh Scattering Velocity Measurements, AIAA Journal, Vol. 37, No. 6, pp. 688-694, 1999.

${ }^{8}$ Bivolaru, D., Danehy, P.M., Grinstead, K. D., Tedder S., Cutler A. D., "Simultaneous CARS and Interferometric Rayleigh Scattering," AIAA Aerodynamic Measurement Technology and Ground Testing Conference, AIAA-2006-2968, San Francisco, June 2006.
} 
${ }^{9}$ A. D. Cutler, G. Magnotti, R. Baurle, D. Bivolaru, S.Tedder, and P. M. Danehy,, "Development of Supersonic Combustion Experiments for CFD Modeling " AIAA Paper 2007-0000, 45th AIAA Aerospace Sciences Meeting and Exhibit, Reno, Nevada, Jan. 8-11, 2007.

${ }^{10}$ Eckbreth, A.C., Laser Diagnostics for Combustion Temperature and Species, $2^{\text {nd }}$ Ed., Gordon and Breach Pub., 1996.

${ }^{11}$ D. Bivolaru, P. M. Danehy, J. W. Lee and R. Gaffney, A. D. Cutler, "Single-Pulse Multi-point Multi-Component

Interferometric Rayleigh Scattering Velocimeter" AIAA Paper 2006-836 44th AIAA Aerospace Sciences Meeting and Exhibit, Reno, Nevada, Jan. 9-12, 2006.

${ }^{12}$ W. D. Kulatilaka, R. P. Lucht, S. F. Hanna, V.R. Katta, "Two-color, two-photon laser-induced polarization spectroscopy (LIPS) measurements of atomic hydrogen in near-adiabatic, atmospheric pressure hydrogen/air flames" Combustion and Flame, 137 (4): 523-537 June 2004.

${ }^{13}$ S.O'Byrne, P.M. Danehy, A. D. Cutler, " $\mathrm{N}_{2} / \mathrm{O}_{2} / \mathrm{H}_{2}$ Dual-pump CARS: Validation experiments", $20^{\text {th }}$ International Congress in Aerospace Simulation Facilities, Goettingen, Germany, August 2003.

${ }^{14}$ A. Mielke, K. Elam, C. Sung, "Rayleigh Scattering Diagnostic for Measurement of Temperature, Velocity, and Density Fluctuation Spectra," 44th AIAA Aerospace Sciences Meeting and Exhibit, AIAA-2006-837, Reno, Nevada, January 9-12, 2006. 